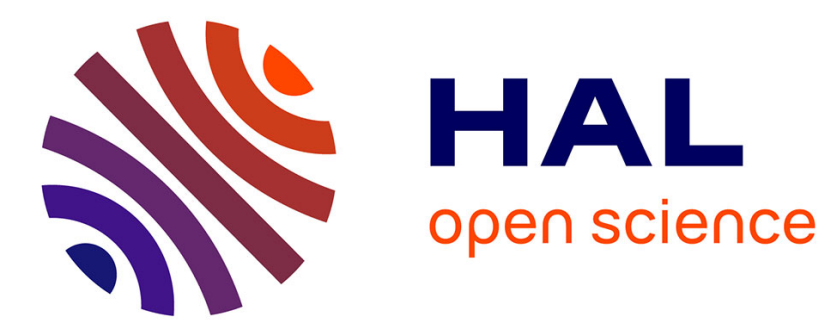

\title{
Discovering keys in RDF/OWL dataset with KD2R
}

\author{
Danai Symeonidou, Nathalie Pernelle, Fatiha Saïs
}

\section{To cite this version:}

Danai Symeonidou, Nathalie Pernelle, Fatiha Saïs. Discovering keys in RDF/OWL dataset with KD2R. In the proceedings of the 2nd International workshop on Open Data (WOD 2013), Jun 2013, Paris, France. 10.1145/2500410.2500419 . hal-01276594

\section{HAL Id: hal-01276594 https://hal.inria.fr/hal-01276594}

Submitted on 19 Feb 2016

HAL is a multi-disciplinary open access archive for the deposit and dissemination of scientific research documents, whether they are published or not. The documents may come from teaching and research institutions in France or abroad, or from public or private research centers.
L'archive ouverte pluridisciplinaire HAL, est destinée au dépôt et à la diffusion de documents scientifiques de niveau recherche, publiés ou non, émanant des établissements d'enseignement et de recherche français ou étrangers, des laboratoires publics ou privés. 


\section{Discovering Keys in RDF/OWL Dataset with KD2R ${ }^{*}$}

\author{
Danai Symeonidou \\ LRI, Université Paris-Sud \\ PCRI, Bat 650 \\ 91405 Orsay, France \\ danai.symeonidou@Iri.fr
}

\author{
Nathalie Pernelle \\ LRI, Université Paris-Sud \\ PCRI, Bat 650 \\ 91405 Orsay, France \\ nathalie.pernelle@Iri.fr
}

\author{
Fatiha Saïs \\ LRI, Université Paris-Sud \\ PCRI, Bat 650 \\ 91405 Orsay, France \\ fatiha.sais@Iri.fr
}

\begin{abstract}
KD2R allows the automatic discovery of composite key constraints in RDF data sources that conform to a given ontology. We consider data sources for which the Unique Name Assumption is fulfilled. KD2R allows this discovery without having to scan all the data. Indeed, the proposed system looks for maximal non keys and derives minimal keys from this set of non keys. KD2R has been tested on several datasets available on the web of data and it has obtained promising results when the discovered keys are used to link data. In the demo, we will demonstrate the functionality of our tool and we will show on several datasets that the keys can be used in a datalinking tool.
\end{abstract}

\section{INTRODUCTION}

Establishing identity links between data items allows crawlers, browsers and applications to combine information from different RDF sources. Many approaches aim to detect sameAs links between data items (see [3] for a survey). Most of these approaches use either specific expert rules that specify conditions that two data items must fulfill in order to be linked $[5,9,1]$ or keys that are declared in the ontology [7]. Nevertheless, when the data are numerous and heterogeneous, these rules or keys cannot easily be specified by a human expert. In $[4,6]$ linkage rules are learnt on a set of reference links while some other approaches focus on key discovery [2, 8].

We present an extension of KD2R [8], an automatic tool for

*Permission to make digital or hard copies of part or all of this work for personal or classroom use is granted without fee provided that copies are not made or distributed for profit or commercial advantage and that copies bear this notice and the full citation on the first page. Copyrights for thirdparty components of this work must be honored. For all other uses, contact the Owner/Author. Copyright is held by the owner/author(s). WOD '13, Jun 03-03 2013, Paris, France ACM 978-1-4503-2020-7/13/06.

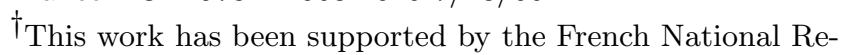
search Agency (ANR) in the setting of the Qualinca project. key discovery in RDF data sources that conform to OWL ontologies. This tool aims to discover key constraints that are composed of several properties. Indeed, non composite keys (e.g. ISBN for books) are rare. KD2R discover keys from datasets where different URIs refer to different world entities (i.e. Unique Name Assumption). Since we work under the Open World Assumption (OWA), KD2R uses heuristics to interpret the absence of information. To avoid scanning all the data, KD2R discovers first maximal non keys before inferring the keys. Indeed, finding two instances that share the same values for the considered set of property expressions suffice to be sure that this set is a non-key. Furthermore, it exploits key inheritance between classes in order to prune the non key search space. This tool has been evaluated on different data sets. In particular, we can show that when a linking tool exploits these automatically discovered keys, relevant identity links can be generated.

\section{KD2R SYSTEM}

Since RDF data sources might contain descriptions that are incomplete (Open World Assumption), a key cannot be deduced from a RDF dataset. Heuristics are needed to declare that a key is valid for a dataset.

\subsection{Optimistic and Pessimistic heuristics}

We consider that a set of property expressions is a key for a class in a data source if for all pairs of distinct instances of this class, there exists a property expression in this set such that all the values are distinct (objects or literal values). We consider that a set of property expressions is a non-key for a class if there exist two distinct instances of this class that share at least one value for all the property expressions of this set. Some combinations of property expressions are neither keys nor non keys: set of property expressions is called an undetermined key for a class if it is not a non-key and there exist two instances of the class such that the instances share the same values for a subset of the property expressions, and the remaining property expressions are unknown for at least one of the two instances.

Distinguishing undetermined keys from keys and non keys allows us to use them differently. Using a pessimistic heuristic, the property for which no value is given can take all the values that appear in the data source. Therefore, the undetermined keys will not be considered as keys. Using an optimistic heuristic, we consider that the not given property values are different from all the values that appear in the data source for this property. This leads to consider the 
undetermined keys as keys.

\subsection{KD2R main steps}

In Figure 1 we show the main steps of KD2R system. The user gives an ontology and a set of data sources. Then, the tool uses the pessimistic or optimistic heuristic to discover the key constraints for each RDF data source independently. In each data source, KD2R is applied on the classes in topologically sorted order. This way, the keys that are discovered in the superclasses are exploited in the processing of their subclasses. For a given data source $s_{i}$ and a given class $c$ we apply Key-Finder which aims at finding keys for the class $c$ that are valid in the data source $s_{i}$. Key-Finder starts by building a prefix tree for this class to represent its instances (see Fig. 1). Using this representation the sets of maximal undetermined keys and maximal non keys are computed. These sets of undetermined keys and non-keys, are used to derive the set of minimal keys. The obtained keys are then merged in order to compute the set of key constraints that are valid for both data sources (see Fig. 2).

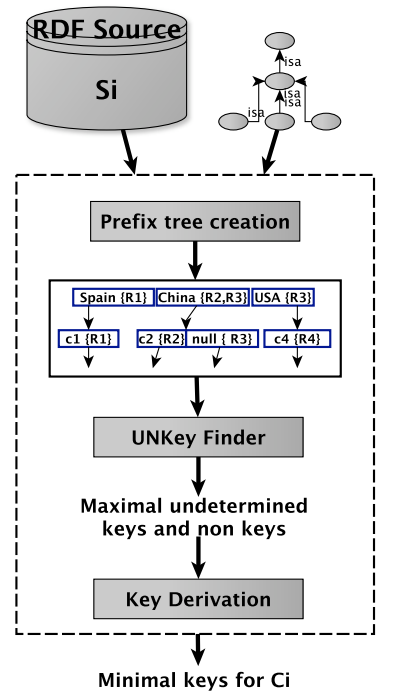

Figure 1: Key discovery for one data source

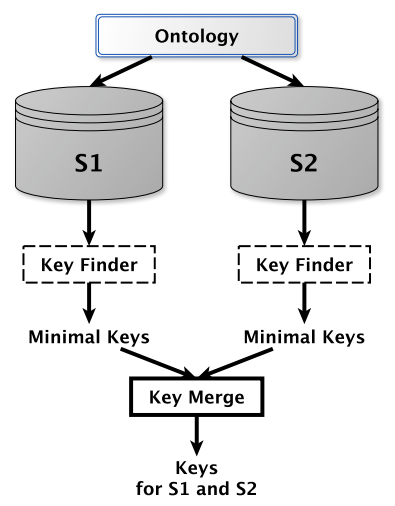

Figure 2: Key merge for two data sources

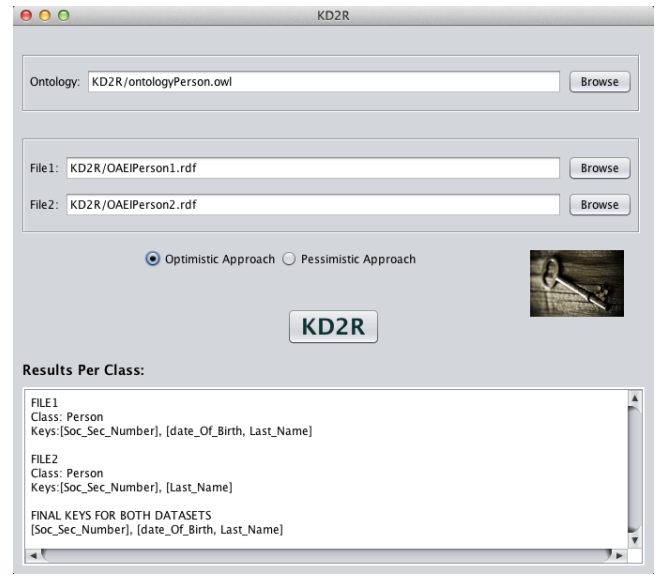

Figure 3: KD2R Graphical User Interface

\section{DEMONSTRATION}

We will use several datasets to show the keys that can be found using pessimistic or optimistic heuristics. Then, the datalinking tool called N2R [7] will be applied on datasets for which a gold standard is available. Thus we will show and compare results that can be odtained when no keys are available (all the properties are used the same way and agregated by an average function), when expert keys are available and when KD2R keys are available.

\section{REFERENCES}

[1] A. Arasu, C. Ré, and D. Suciu. Large-scale deduplication with constraints using dedupalog. In ICDE, pages 952-963, 2009.

[2] M. Atencia, J. David, and F. Scharffe. Keys and pseudo-keys detection for web datasets cleansing and interlinking. In $E K A W$, pages 144-153, 2012.

[3] A. Ferrara, A. Nikolov, and F. Scharffe. Data linking for the semantic web. Int. J. Semantic Web Inf. Syst., 7(3):46-76, 2011.

[4] R. Isele and C. Bizer. Learning expressive linkage rules using genetic programming. PVLDB, 5(11):1638-1649, 2012.

[5] W. L. Low, M. L. Lee, and T. W. Ling. A knowledge-based approach for duplicate elimination in data cleaning. Information Systems, 26:585-606, 2001.

[6] A.-C. N. Ngomo and K. Lyko. Eagle: Efficient active learning of link specifications using genetic programming. In 9th Extended Semantic Web Conference (ESWC), pages 149-163, 2012.

[7] F. Saïs, N. Pernelle, and M.-C. Rousset. Combining a logical and a numerical method for data reconciliation. Journal on Data Semantics, 12:66-94, 2009.

[8] D. Symeonidou, N. Pernelle, and F. Saïs. Kd2r: A key discovery method for semantic reference reconciliation. In OTM Workshops, pages 392-401, 2011.

[9] J. Volz, C. Bizer, M. Gaedke, and G. Kobilarov. Discovering and maintaining links on the web of data. In Proceedings ISWC'09, pages 650-665. Springer-Verlag, 2009. 